\section{A laboratory-based study of COVID-19 in Casablanca, Morocco}

Idrissa Diawara, ${ }^{1,2}$ Houda Benrahma, 2,3 Meskaouni Nida, ${ }^{2}$ Jalila Rahoui, ${ }^{2}$

Fatima-Zahra Moujid, ${ }^{2}$ Khadija Jaras, ${ }^{2}$ Rachid Benmessaoud, ${ }^{2}$ Khadija Arouro, ${ }^{2}$ Zahra Aadam, ${ }^{2}$ Salma Nahir, ${ }^{2}$ Zineb

Aouzal, ${ }^{2}$ Hajar Elguezzar, ${ }^{2}$ Leila Jeddan, ${ }^{2}$ Hind Rida, ${ }^{2}$ Fadoua Ousti, ${ }^{2}$ Jalila El Bakkouri, ${ }^{2}$ Imane Smyej, ${ }^{2}$ Chakib Nejjari ${ }^{4}$

${ }^{1}$ Department of Health Techniques, Faculty of Nursing and Allied Health Sciences; ${ }^{2}$ National Reference

Laboratory; ${ }^{3}$ Faculty of Medicine; ${ }^{4}$ International School of Public Health, Mohammed VI University of Health Sciences (UM6SS), Casablanca, Morocco

\section{Abstract}

Given the spread of coronavirus disease 2019 (COVID-19) and its impact on human health, laboratory confirmation of diagnosis is essential. This study examined the contribution of laboratory diagnosis to the detection of severe acute respiratory syndrome coronavirus 2 (SARS-CoV-2) in the diagnosis of COVID-19, taking into account patient risk of exposure to SARS-CoV-2, clinical symptoms and comorbidities. A cross-sectional, laboratory-based study was carried out from 1 April 2020 to 30 April 2020 at the National Reference Laboratory in Morocco using nasopharyngeal samples from patients admitted to the Cheikh Khalifa International University Hospital or other hospitals in Casablanca. A one-step reverse transcription real-time polymerase chain reaction (RT-PCR) was used to detect the presence of the SARS-CoV-2 genome. A national epidemiological investigation form was used to analyze patient exposure risk, clinical symptoms and comorbidities. A total of 793 samples from 375 patients were analyzed and 1150 RT-PCR tests were conducted; 116 patients $(30.93 \%)$ were COVID-19 positive. Travel to a risk zone, contact with a confirmed COVID-19 case and contact with a person who had been in a risk zone were significantly associated with being positive for COVID-19. Fever and cough were the main symptoms; $7.76 \%$ of positive patients were asymptomatic. This is the first laboratory-based study in Morocco for the diagnosis of COVID-19. Laboratory diagnosis of COVID-19 by RTPCR associated with knowledge of expo- sure risk factors and clinical symptoms and comorbidities remains essential for clinicians for early, appropriate medical management COVID-19 patients.

\section{Introduction}

In mid-December 2019, an epidemic of a viral respiratory disease, officially named by the World Health Organization (WHO) as coronavirus disease 2019 (COVID-19) caused by severe acute respiratory syndrome coronavirus 2 (SARS-CoV-2), was discovered in the city of Wuhan, Hubei province, China. This epidemic then spread to the rest of the world, prompting WHO to declare a public health emergency of international concern and subsequently to declare the COVID-19 outbreak a pandemic. ${ }^{1,2}$

Since the start of COVID-19 in December 2019 to April 30, 2020, a total 3,090,445 confirmed cases of COVID-19 have been reported worldwide, with 217,769 deaths. As of April 30, 2020, there were 182,417 confirmed cases in the Eastern Mediterranean region with 7447 deaths. In the WHO African region, a total of 24,713 confirmed cases with 938 deaths were reported. In Morocco in April 30, 2020 , there were 4359 confirmed cases with 168 deaths was reported. ${ }^{1,3}$ At present, there are no specific antiviral drugs or vaccine against COVID-19 infection for potential therapy of humans. Studies have shown that broad-spectrum antivirals like remdesivir, oseltamivir, lopinavir, ritonavir, ganciclovir and the antiparasitic chloroquine could be highly effective in the control of COVID$19.4,5$

Coronaviruses are a large family of viruses that can be pathogenic in humans and animals. The viruses of the Coronaviridae family are enveloped viruses with a single-stranded RNA genome of positive polarity. ${ }^{6}$ Recently, Three types of coronaviruses, which are not found naturally in humans but in mammals, have been discovered and have been the origin of serious lung infections (viral pneumonia): SARS-CoV, Middle East Respiratory Syndrome Coronavirus (MERS-CoV) and SARS-CoV-2, responsible respectively for Severe Acute Respiratory Syndrome (SARS), Middle East Respiratory Syndrome (MERS) and COVID-19.4,7-9 For the last coronavirus responsible for COVID-19, the most common symptoms are fever, fatigue and a dry cough. Some patients experience pain, nasal congestion, runny nose, sore throat or diarrhea. About one in six people with the disease have more severe symptoms, including dyspnea.
Correspondence: Idrissa Diawara, Department of Health Techniques, Faculty of Nursing and Allied Health Sciences, Mohammed VI University of Health Sciences (UM6SS), Anfa City, Bld Mohammed Taïeb Naciri, Commune Hay Hassani, 82403 Casablanca, Morocco. E-mail: idiawara@um6ss.ma

Key words: COVID-19; SARS-CoV-2; risk factors; laboratory test; RT-PCR.

Contributions: The conception and design of the study were carried out by: ID, HB, NM, $\mathrm{JR}, \mathrm{CN}$. Acquisition of data was made by: FZM, KJ, RB, KA, ZA, SN, ZA, HE, LJ and HR. Analysis and interpretation of data were made by: ID, HB, NM, JR, FO, JEB and IS. Drafting the article and the critically revising for important intellectual content were made by: ID, HB, NM and CN. Final approval of the version to be submitted were done by: ID, HB, $\mathrm{NM}$ and $\mathrm{CN}$.

Conflict of interest: The authors declare conflicts of interest.

Availability of data and materials: All data generated or analyzed during this study are included in this published article.

Ethics approval and consent to participate: This study was approved by the Ethical Committee for Biomedical Research of Mohammed VI University of Health Sciences, Casablanca, Morocco (Ref: CE_UM6SS/2/06/2020 - May 4, 2020). The study is conformed with the Helsinki Declaration of 1964, as revised in 2013, concerning human and animal rights. The patients or legal guardians were informed in writing about the study and approved and consented to the use of their data for scientific purposes.

Informed consent: Written informed consent was obtained from a legally authorized representative(s) for anonymized patient information to be published in this article.

Received for publication: 3 June 2021. Revision received: 14 September 2021

Accepted for publication: 30 September 2021.

This work is licensed under a Creative Commons Attribution NonCommercial 4.0 License (CC BY-NC 4.0).

(C) Copyright: the Author(s),2021

Licensee PAGEPress, Italy

Journal of Public Health in Africa 2021:12:1458 doi:10.4081/jphia.2021.1458

Seniors and those with other health conditions (high blood pressure, heart problems or diabetes) are more likely to have serious symptoms. ${ }^{9,10}$

Currently, the diagnosis of SARS-CoV- 
2 infection is a public health issue. SARSCoV-2 screening allows the diagnosis of COVID-19 infection. This screening is based on the detection of the RNA of SARS-CoV-2 by a reverse transcriptase PCR technique in real time. These PCR assays specifically detect specific genes of SARS-CoV-2. The most relevant targets are: the envelope ( $E$ gene) gene, the RNA dependent RNA polymerase ( $R d R p$ gene) and the nucleocapsid ( $N$ gene) and the ORF1ab encoding proteins for RNA replication (ORFlab gene). ${ }^{11}$ Alongside the specific RT-PCR, the diagnosis of SARS-CoV2 infection may also be made by the determination of $\mathrm{IgM} / \mathrm{IgG}$ in serum, ${ }^{12}$ In Morocco several laboratories have been authorized by the Ministry of Health to carry out these diagnostic tests. Among these laboratories, the National Reference Laboratory (Laboratoire national de reference, LNR) attached to the Mohammed VI University of Health Sciences is an advanced laboratory equipped with several molecular diagnostic platforms, including those for COVID-19.

In Morocco, there are no data on laboratory-based studies of the management of COVID-19. Given the spread of the new coronavirus and its impact on human health, laboratory confirmation of diagnosis is essential. This study aims to provide the contribution of RT-PCR to the detection of SARS-CoV-2 in the diagnosis of COVID19 from patients taking into account the patient exposition risk to SARS-CoV-2, clinical symptoms and comorbidities.

\section{Materials and Methods}

\section{Ethical considerations}

This study was approved by the Ethical Committee for Biomedical Research of Mohammed VI University of Health
Sciences, Casablanca, Morocco (Ref: CE_UM6SS/2/06/2020 - May 4, 2020). The patients or legal guardians were informed in writing about the study and approved and consented to the use of their data for scientific purposes. The data were systematically entered in the LNR system database guaranteed by a restricted access code, and the study was carried out confidentially and anonymously.

\section{Patients, sampling and study design}

From 1 April 2020 to 30 April 2020,we carried out a cross-sectional, laboratorybased study that included samples from patients admitted to the Cheikh Khalifa International University Hospital or from to other hospitals in Casablanca, Morocco. All samples were collected for COVID-19 diagnosis. Sampling was done using nasopharyngeal swab conserved in Universal Transport Media (UTM) for viruses. The collected swabs were placed into $3 \mathrm{~mL}$ of UTM and sent to the LNR in a triple packaging system at $+4^{\circ} \mathrm{C}$. All laboratory procedures were conducted at the LNR.

To study the exposure risk factors to SARS-CoV-2, clinical symptoms, and comorbidities, we used the national epidemiological investigation form for possible cases of COVID-19 provided by the Moroccan Ministry of Health. All samples were accompanied by this form. We investigated data related to: i) identity (gender, age, sex and address), ii) risk factors for exposure to COVID-19 (travel to a risk zone, contact with a confirmed case, attendance at a hospital structure, contact with a person who had visited a risk zone, and is a patient of a healthcare professional working in the hospital or in a laboratory realizing COVID-19 diagnostic test, iii) clinical symptoms (start date of symptoms, fever, cough, sore throat, breathing difficulty, headache, myalgia, diarrhea, abdominal pain, vomiting, respiratory distress and other non-specific symptoms), iv) co-morbidities and risk factors associated with COVID-19 (diabetes, high blood pressure, chronic respiratory disease, pregnancy, immunosuppression and other non-specific comorbidity). All samples sent to LNR without the completed form were excluded from the study.

\section{RNA extraction and RT-PCR SARS- CoV2 detection}

For RNA extraction, we used AccuPrep $^{\circledR}$ Viral RNA Extraction Kit (Bioneer Corporation, Munpyeongseo-ro, Daedeok-gu, Daejeon, Republic of Korea) according to the manufacturer's instructions. A one-step reverse transcription realtime polymerase chain reaction (RT-PCR) was used to detect the presence of the SARS-CoV-2 genome by amplifying specific $R d R p, E$, and $N$ genes using GeneFinder $^{\text {TM }}$ COVID-19 Plus RealAmp Kit according to the manufacturer's protocol (OSANG Healthcare Co., Ltd, Anyangcheondong-ro, Dongan-gu, Anyang-si, Gyeonggi-do, Korea). All RTPCR assays were performed on a CFX96 Real-Time System (Bio-Rad, Hercules, California, United States).

For test interpretation, we used two recommendations: i) the recommendations of GeneFinder $^{\mathrm{TM}}$ COVID-19 Plus RealAmp Kit according to the manufacturer's protocol, ii) recommendations for the interpretation of laboratory tests and recommendations for laboratories investigating SARS published by WHO (Table 1). ${ }^{13}$

\section{The laboratory workflow for the confirmation of COVID-19 cases}

For confirmation of COVID-19 cases, our laboratory used validated commercial kits that detect three targets of SARS-CoV2 genome. Of these three targets, $N$ and $R d R p$ genes are the most specific to SARS-

Table 1. Results interpretation of GeneFinderTM COVID-19 Plus RealAmp Kit according to the manufacturer recommendations (March-2020 “rev.2” IFMR-45).

\begin{tabular}{|c|c|c|c|c|}
\hline \multirow[t]{2}{*}{ Results of RT-PCR } & \multicolumn{4}{|c|}{ Ct range } \\
\hline & RdRp & E & $\mathbf{N}$ & IC \\
\hline COVID-19 Positive & $\begin{array}{l}\leq 43 \\
\leq 43 \\
\leq 43\end{array}$ & $\begin{array}{l}\leq 43 \\
\leq 43 \\
\text { U.D }\end{array}$ & $\begin{array}{l}\leq 43 \\
\text { U.D } \\
\leq 43\end{array}$ & $\begin{array}{l}\leq 35 \\
\leq 35 \\
\leq 35\end{array}$ \\
\hline COVID-19 Positive if $R d R p \leq 43$ after the second test & $\leq 43$ & U.D & U.D & $\leq 35$ \\
\hline COVID-19 Positive if $E$ and $N \leq 43$ after the second test & U.D & $\leq 43$ & $\leq 43$ & $\leq 35$ \\
\hline COVID-19 Positive if $N \leq 43$ after the second test & U.D & U.D & $\leq 43$ & $\leq 35$ \\
\hline Beta coronavirus & U.D & $\leq 43$ & U.D & $\leq 35$ \\
\hline Negative & U.D & U.D & U.D & $\leq 35$ \\
\hline
\end{tabular}

Ct: Cycle Threshold; RNA dependent RNA polymerase (RdRp gene) gene; Envelope (E gene) gene; Nucleocapsid (N gene) gene; IC: internal control; U.D= undetermined. 
CoV-2. Therefore, the presence of one and/or the two genes are sufficient the confirmed a COVID-19 cases. To avoid samples and PCR contamination, with the Biological Safety Levels 3 environment for RNA extraction and handling primary samples from suspect patients, all the further steps were handled separately: oligonucleotides and PCR mastermix reagents were handled in one room under a PCR hood with specified laboratory coats. Addition of sample RNA to the mastermix took place in a second room. The third room is the machine room to launch the PCR on CFX96 Thermal cycler. This workflow takes into account of international standards for the organization of laboratories practicing the diagnosis of covid-19. ${ }^{11-13}$

\section{Statistical analysis}

Anonymous computerized data were analyzed by EpiInfo 7 (Centers for Disease Control, Atlanta, Georgia, United States) and Microsoft Excel (Microsoft, Morocco). Qualitative variables were analyses by performing non-parametric tests: The chi square test or Fisher's exact test or Yates correction test. The estimation of associations between COVID-19 and exposure risk factors, clinical symptoms and comorbidities were assessed by calculating Odd Ratios (OR) or Risk Ratios (RR). Differences were considered significant if the p-value was $<0.05$. Statistical signifi- cance was determined with 95\% Confidence Internals (CI).

\section{Results}

During the study period, 793 samples were analyzed by RT-PCR from 375 patients. A total of 1150 RT-PCR tests were completed with a range of 1 to 3 tests per patient. Among the 375 patients, RT-PCR for the detection of SARS-CoV-2 was positive in 116 patients $(30.93 \%)$. The sex ratio (Males/Females) of positive patients was 1.37 with an overall average age of $47 \pm 20$ years old (aged from 5 to 84 years).

Of all the exposure risk factors studied, travel to a risk zone, contact with a confirmed COVID-19 case and the contact with a person who has sojourned in a risk zone were significantly associated to the COVID-19 (Table 2). The main factor strongly associated with contracting COVID-19 was contact with a confirmed COVID-19 case. The risk factors relative to a risk zone were a country where an epidemic was declared or a national region where a high concentration of cases was detected. As for the clinical symptoms of COVID-19 positive patients, the average time from symptom onset to the confirmation of COVID-19 by RT-PCR at the laboratory was $14.13 \pm 6.61$ days (range: 3 to 30 days).
The main statistically significant symptoms of COVID-19-positive patients were fever and cough (Table 3 ). For patients with fever, the average temperature was $38.3 \pm 0.39^{\circ} \mathrm{C}$. A total of $7.76 \%$ of positive patients ( 9 patients) were asymptomatic. Moreover, several other nonspecific symptoms were associated with COVID-19-positive patients. Mainly, $8.62 \%$ of patients had anosmia, 4.31\% had ageusia and $2.58 \%$ had dizziness. Other nonspecific symptoms described among less than one percent of patients were anorexia, dyspnea, respiratory distress, nausea, vomiting and anemia.

In our study, age over 60 years was a statistically significant comorbidity factor (accounting for $38.83 \%$ of patients; $\mathrm{RR}=1.44, \quad 95 \% \quad \mathrm{CI}=1.055-1.969, \quad \mathrm{p}-$ value $=0.02$ ) associated with COVID-19 (Table 4). Other comorbidities that could potentially be associated with COVID-19 were presence of diabetes $(12.05 \%$ of positive patients) and high blood pressure (19.28\% of positive patients).

\section{Discussion}

Since the start of outbreak in December 2019, the SARS-CoV-2 virus has continued to spread worldwide. ${ }^{3}$ The widespread distribution of this virus, more than 213 countries and territories, has led to a major concern, globally. ${ }^{14-15}$ After MERS-CoV and

Table 2. Exposure risk factors to COVID-19 of positive patients admitted to the Cheikh Khalifa International University Hospital in Casablanca, Morocco, from $1^{\text {st }}$ to April $30^{\text {th }} 2020$.

\begin{tabular}{|c|c|c|c|c|}
\hline Risk factors exposure to COVID-19 & Percentage of COVID-19 positive patients & Risk ratio & CI $95 \%$ & P-value \\
\hline Travel to a risk zone & 14.29 & 2.29 & $1.347-6.395$ & 0.004 \\
\hline Contact with a confirmed COVID-19 case & 66.67 & 1.55 & $1.255-1.922$ & 0.0002 \\
\hline Attendance at a hospital structure & 14.29 & 0.5 & $0.287-0.886$ & 0.01 \\
\hline Contact with a person who has sojourned in a risk zone & 29.76 & 1.81 & $1.169-2.826$ & 0.008 \\
\hline Is the patient a healthcare professional working in the & 5.95 & 0.43 & $0.174-1.079$ & 0.05 \\
\hline
\end{tabular}

or in a laboratory realizing COVID-19 diagnostic test?

CI: confidence interval.

Table 3. Clinical symptoms of covid-19 positive patients admitted to the Cheikh Khalifa International University Hospital in Casablanca, Morocco, from $1^{\text {st }}$ to April $30^{\text {th }} 2020$.

\begin{tabular}{lcccc} 
Clinical symptoms & Percentage of COVID-19 positive patients & Odd Ratio & CI 95\% & P-value \\
Fever & 47.44 & 2.66 & $1.553-4.554$ & 0.0002 \\
Cough & 53.86 & 2.36 & $1.397-4.003$ & 0.001 \\
\hline Sore throat & 20.51 & 0.93 & $0.4925-1,757$ & 0.823 \\
Breathing difficulty & 23.08 & 0.66 & $0.363-1.203$ & 0.173 \\
\hline Headache & 37.18 & 1.48 & $0.861-2.558$ & 0.153 \\
Myalgia & 30.77 & 1.37 & $0.777-2.431$ & 0.273 \\
\hline Diarrhea & 19.23 & 1.45 & $0.739-2.878$ & 0.273 \\
Abdominal pain & 12.82 & 1.01 & $0.468-2.196$ & 0.972 \\
\hline Vomiting & 11.54 & 1.24 & $0.543-2.842$ & 0.61 \\
\hline
\end{tabular}

CI: confidence interval. 
Table 4. Comorbidities factors of COVID-19 positive patients admitted to the Cheikh Khalifa International University Hospital in Casablanca, Morocco, from $1^{\text {st }}$ to April $30^{\text {th }} 2020$.

\begin{tabular}{|c|c|c|c|c|}
\hline Comorbidities factors & Percentage of COVID-19 patients & Risk Ratio & CI $95 \%$ a & P-value \\
\hline Diabetes & 12.05 & 0.78 & $0.405-1.513$ & 0.46 \\
\hline High blood pressure & 19.28 & 1.15 & $0.678-1.955$ & 0.6 \\
\hline Chronic respiratory disease & 4.82 & 0.39 & $0.142-1.093$ & 0.05 \\
\hline Pregnancy & 1.2 & 1.33 & $0.122-14.488$ & 0.81 \\
\hline Immunosuppression & 1.2 & 0.89 & $0.094-8.413$ & 0.92 \\
\hline Age over 60 years old & 38.83 & 1.44 & $1.055-1.969$ & 0.02 \\
\hline
\end{tabular}

${ }^{\mathrm{a}} \mathrm{Cl}$ : confidence interval.

SARS-CoV, the SARS-CoV-2 virus is the third contagious coronavirus leading to an epidemic in the 21 st century. ${ }^{9}$ the objective of the current study was to provide the contribution of PCR to the detection of SARSCoV-2 in the diagnosis of COVID-19 from patients taking into account of the patient exposition risk to SARS-CoV-2, clinical symptoms and comorbidities. Laboratory diagnosis is necessary because it mainly distinguished COVID-19 from other known viruses of pneumonia and from others noninfectious diseases. Identification of COVID-19 includes virus isolation and/or viral nucleic acid detection. In the present study, for only month of April, we realized more than 1150 RT-PCR tests. The overall prevalence of COVID-19 was 30.93\% taking into account our inclusion criteria. The investigation of exposure risk factors to COVID-19 of our study revealed that: travel to a risk zone, contact with a confirmed case and contact with a person who has been in a risk zone were the major risk factors exposure to COVID-19. This is also well described in the WHO suspect, probable and confirmed COVID-19 cases, as well as contact cases definitions, the risk factors found in our study constitute some of the main factors that contribute to the contamination of SARS-CoV2 and to developing COVID-19. ${ }^{3}$

The average time from symptoms onset to the confirmation of COVID-19 by RTPCR at the laboratory was $14.13 \pm 6.61$ days and ranged from 3 to 30 days. The mean incubation periods of COVID-19 in recent publications have differed slightly. Lauer et $a l$., with 181 cases with confirmed SARSCoV-2 infection detected outside Hubei province in China before 24 February 2020 , reported that the median incubation period for these cases was 5.5 days (CI, 4.4 to 7.0 days). ${ }^{16}$ Wang et al., in Wuhan, China with 138 cases, reported that the median durations from first symptoms range to were 5 days to 8 days. ${ }^{17}$ In summary, the incubation means ranged from 3 days to 14 days with an average CI of 0 to 30 days. ${ }^{10,16,17}$

Importantly, $7.76 \%$ of positive patients
(9 patients) were asymptomatic. Elsewhere, the Epidemiology Working Group for NCIP Epidemic Response of the Chinese Center for Disease Control and Prevention, with a total of 72,314 patients, reported that there were 889 asymptomatic cases $(1.2 \%)$, and $44,672(61.8 \%)$ confirmed cases among the total number of patients. ${ }^{18}$ They had no symptoms upon confirmation of diagnosis of COVID-19 by RT-PCR. As reported elsewhere, ${ }^{19,20}$ these asymptomatic patients constitute a big challenge for the management of COVID-19, because asymptomatic patients play a key role in the spread of virus.

The main significant symptoms associated with COVID-19 in our study were fever and cough. For patients with fever, the average temperature was $38.3 \pm 0.39$. Patient age over 60 years was a significant comorbidity factor associated with the COVID-19 followed by diabetes and patients with high blood pressure. Out of these findings, several studies reported common clinical symptoms include fever, cough, myalgia, upper respiratory tract symptoms like rhinorrhea, sneezing, and sore throat, and in addition to the increasing dyspnea with hypoxemia. Some patients infected with COVID-19 developed gastrointestinal symptoms like diarrhea. ${ }^{4,21}$ Around the world, the most common symptoms were fever (98\%), cough $(76 \%)$, myalgia or fatigue $(44 \%)$; and atypical symptoms included sputum (28\%), headache (8\%), hemoptysis $(5 \%)$ and diarrhea $(3 \%) .{ }^{10,21}$ As already reported, many underlying diseases (comorbidities) increase the risk of patients with COVID19, including particularly elderly patient, diabetes, hypertension, but also cardiovascular disease. ${ }^{21}$

\section{Limitations}

Because this study focused on laboratory-reported cases of COVID-19 and RTPCR analysis, the evolution of clinical and biological parameters of patients was not assessed.

\section{Conclusions}

This is the first laboratory-based study in Morocco for the diagnosis of COVID-19. Through this study, we concluded that the contribution of laboratory for the diagnosis of COVID-19 cases by RT-PCR is essential for management of patients with COVID19. We also argued that laboratory tests associated to the knowledge of exposure risk factors, clinical symptoms and comorbidities remains determining for clinicians in the early appropriate medical management of patients with COVID-19.

\section{References}

1. WHO. World Health Organization : COVID-19 DISEASE 2019 (COVID19). WHO, 2020a. Accessed: 10 April 2020. Available from: https:/www.who.int/fr/emergencies/dis eases/novel-coronavirus-2019/advicefor-public/q-a-coronaviruses

2. Zhu N, Zhang D, Wang W, et al. A novel coronavirus from patients with pneumonia in China, 2019. New Engl J Med 2020;382:727-33.

3. WHO. World Health Organization, Coronavirus disease 2019 (COVID-19) Situation Report - 101 2020b. Accessed 02 May 2020. Available from: https://www.who.int/docs/defaultsource/coronaviruse/situationreports/20200430-sitrep-101-covid19.pdf

4. Rothan HA, Byrareddy SN. The epidemiology and pathogenesis of coronavirus disease (COVID-19) outbreak. J Autoimm 2020;109:102433.

5. Wang M, Cao R, Zhang L, et al. Remdesivir and chloroquine effectively inhibit the recently emerged novel coronavirus (2019-nCoV) in vitro. Cell Res 2020;30:269-271.

6. Lu R, Zhao X, Li J, et al. Genomic characterisation and epidemiology of 2019 novel coronavirus: implications for 
virus origins and receptor binding. Lancet 2020;395:565-74.

7. Liu P, Shi L, Zhang W, et al. Prevalence and genetic diversity analysis of human coronaviruses among cross-border children. Virol J 2017;14:230.

8. Phan T. Novel coronavirus: From discovery to clinical diagnostics. Infect Genet Evol 2020;79:104211.

9. Guarner J. Three emerging coronaviruses in two decades. Am J Clinical Pathol 2020;153:420-1.

10. $\mathrm{Wu} \mathrm{D,} \mathrm{Wu} \mathrm{T,} \mathrm{Liu} \mathrm{Q,} \mathrm{Yang} \mathrm{Z.} \mathrm{The}$ SARS-CoV-2 outbreak: What we know. International journal of infectious diseases. IJID 2020;94:44-8.

11. Konrad R, Eberle U, Dangel A, et al. Rapid establishment of laboratory diagnostics for the novel coronavirus SARS-CoV-2 in Bavaria, Germany, February 2020. Eurosurveillance 2020;25:2000173.

12. Li Z, Yi Y, Luo X, et al. Development and clinical application of a rapid IgMIgG combined antibody test for SARS-
CoV-2 infection diagnosis. J Med Virol 2020;92:1518-24.

13. WHO. Recours aux méthodes de laboratoire pour le diagnostic du SRAS. WHO 2020c. Accessed: 10 April 2020. Available from: https://www.who.int/csr/sars/labmethodsfr/en/

14. Lai CC, Shih TP, Ko WC, et al. Severe acute respiratory syndrome coronavirus 2 (SARS-CoV-2) and coronavirus disease-2019 (COVID-19): The epidemic and the challenges. Int $\mathrm{J}$ Antimicr Agents 2020;55:105924.

15. Emami A, Javanmardi F, Pirbonyeh N, Akbari A. Prevalence of underlying diseases in hospitalized patients with COVID-19: a systematic review and meta-analysis. Arch Acad Emerg Med 2020;8:e35.

16. Lauer SA, Grantz KH, Bi Q, et al. The incubation period of coronavirus disease 2019 (COVID-19) from publicly reported confirmed cases: estimation and application. Ann Internal Med
2020;172:577-82.

17. Wang $\mathrm{D}, \mathrm{Hu} \mathrm{B}, \mathrm{Hu} \mathrm{C}$, et al. Clinical Characteristics of 138 Hospitalized Patients With 2019 Novel CoronavirusInfected Pneumonia in Wuhan, China. JAMA 2020;323:1061-9.

18. Epidemiology Working Group for NCIP Epidemic Response, Chinese Center for Disease Control and Prevention. [The epidemiological characteristics of an outbreak of 2019 novel coronavirus diseases (COVID-19) in China]. Zhonghua Liu Xing Bing Xue Za Zhi 2020;41:145-51.

19. Bai Y, Yao L, Wei T, et al. Presumed Asymptomatic Carrier Transmission of COVID-19. JAMA 2020;323:1406-7.

20. Yu X, Yang R. COVID-19 transmission through asymptomatic carriers is a challenge to containment. Influenza Other Respir Viruses 2020;14:474-5.

21. Huang C, Wang Y, Li X, et al. Clinical features of patients infected with 2019 novel coronavirus in Wuhan, China. Lancet 2020;395:497-506. 